\begin{tabular}{|c|c|}
\hline \multirow{3}{*}{ 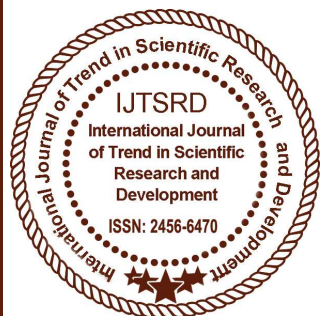 } & $\begin{array}{l}\text { International Journal of Trend in Scientific } \\
\text { Research and Development (IJTSRD) }\end{array}$ \\
\hline & International Open Access Journal \\
\hline & ISSN No: 2456 - 6470 | www.ijtsrd.com | Volume - 2 | Issue - 2 \\
\hline
\end{tabular}

\title{
Mitosis Detection in Phase Contrast Microscopy Image Sequences of Stem Cell Populations: A Critical Review
}

\begin{abstract}
Rakshitha K. S
Masters in Technology, Department of Computer

Science \& Engineering, BMSIT \& M Yelahanka,

Bengaluru, Karnataka, India
\end{abstract}

\author{
Radhika K. R \\ Assistant Professor, Department of Computer \\ Science \& Engineering, BMSIT \& M Yelahanka, \\ Bengaluru, Karnataka, India
}

\begin{abstract}
Modern technology has enabled monitoring of large populations of live cells over extended time periods in experimental settings. Live imaging of cell populations growing in a culture vessel has been widely adopted in biomedical experiments. Such experiments create large amount of time-lapse images, and the information embedded in these images holds tremendous promise for scientific discovery. The growing amount of such data calls for automated or semi-automated computer vision tools to detect and analyze the dynamic cellular processes, and to extract the information necessary for biomedical study. Live cells are often of low contrast with little natural pigmentation, therefore they usually need to be stained or fixed in order to be visible under bright field microscopy or florescence microscopy. However, fixing or staining may destroy the cells or introduce artifacts.

\section{INTRODUCTION}

as cell tracking and lineage construction. The information provided by mitosis detection can be further utilized in biological and medical applications such as cell culture monitoring, stem cell manufacturing, drug development, tissue engineering and so on. Currently, the detection of mitosis is typically performed by people with biological expertise. However, detecting mitosis from large number of cell images by human is both tedious and error prone.

The task of computer vision based mitosis detection can be further categorized into identification, localization and segmentation problems. Given a static cell image or a dynamic cell image sequence, the goal of mitosis identification is to detect whether there is mitosis in the input data. In addition, mitosis localization approaches can further localize the division of cells in mitotic process spatially and temporally from the input sequence.
\end{abstract}

Label-free imaging, in particular phase contrast microscopy, is highly desirable for live cell imaging because it allows cells to be examined in their natural state, therefore enables live cell imaging over extended time periods. In computer vision literature, the detection of cell division is often referred to as mitosis detection. Mitosis detection is essential for quantitative analysis of cell populations. The detection of mitotic cells can provide valuable information to improve the performance of tasks such 


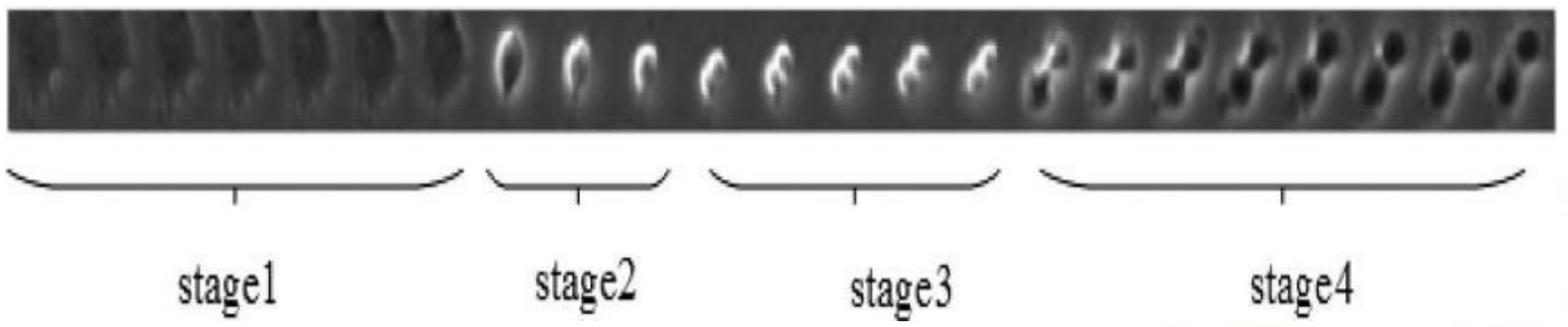

Figure 1.Four stages of visual appearance transition

Based on the changes of visual appearance of stem cells in phase contrast microscopy images, mitosis of stem cells can be segmented into four stages, and each of them is related to biological phases in the cell circle: 1. Interphase stage, when the cells still looks normal in appearance; 2 . Start of mitosis, when the cells begin to shrink, round up and get brighter while going through prophase, metaphase and anaphase; 3 . Formation of daughter cells, when two daughter cells become visible and attached with each other forming the shape ' 8 ' at the period of telophase; 4 . Separation of daughter cells, when two daughter cells get completely sepa-rated after cytokinesis is completed. These four stages in the visual appearance transition are illustrated in fig1.

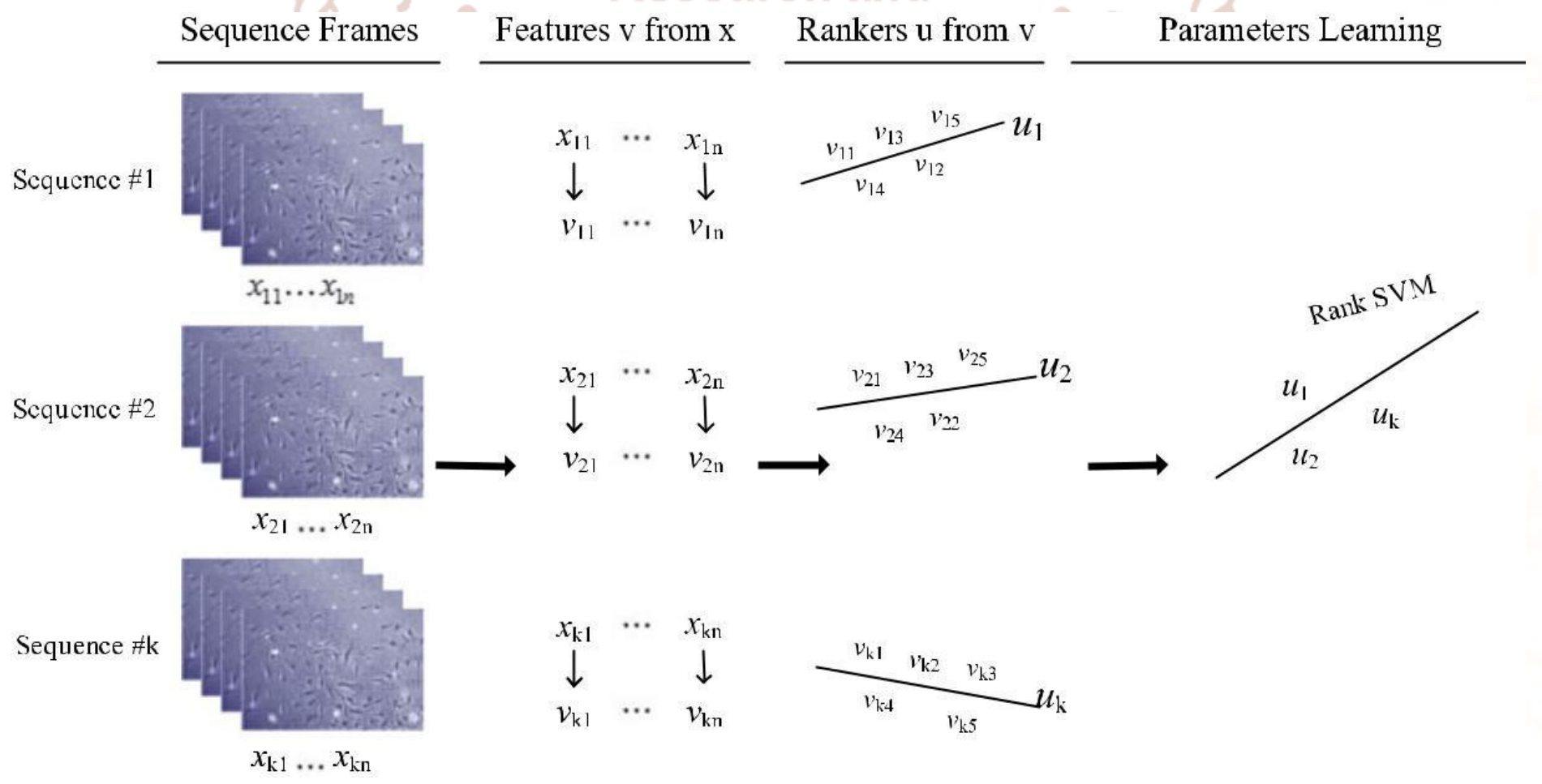

Fig 2: Frame work of our approach

The number of mitotic figures visible in histology sections is an important indicator for cancer screening and assessment. Normally, the count is performed

\section{BACKGROUND AND RELATED WORK:}

State-of-the-art methods for mitotic event detection in microscopy image sequences can be generally classified into the three following categories.

\begin{tabular}{|l|l|l|}
\hline Method & Precision(\%) & Recall(\%) \\
\hline 3D-CNN [11] & 70.84 & 66.39 \\
\hline Schuldt [12] & 74.98 & 71.36 \\
\hline Niebles [14] & 66.33 & 61.04 \\
\hline Dollar [15] & 60.84 & 62.79 \\
\hline Jhuang [16] al & 50.04 & 49.74 \\
\hline
\end{tabular}

Scientific

ch anc 
comparability of results obtained in different labs. Mitosis detection is very hard. In fact, mitosis is a complex process during which a cell nucleus undergoes various transformations. In addition, different image areas are characterized by different tissue types, which exhibit highly variable appearance. A large amount of different structures can be observed in histology images stained with Hematosin \& Eosin, and in particular many dark-blue spots, most of which correspond to cell nuclei.

As an additional complication, in later stages of the mitosis process a nucleus may appear to split in two dark-blue spots, to be counted as one single mitosis. Our approach is conceptually very simple. We use a supervised Deep Neural Network (DNN) as a powerful pixel classifier. The DNN is a max-pooling

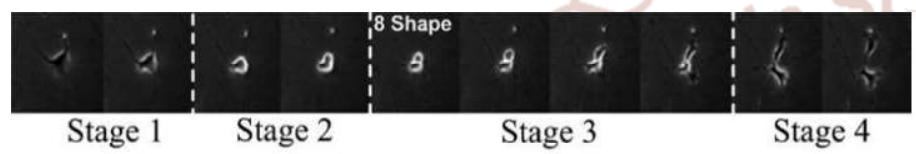

(MP) convolutional neural network (CNN). It directly operates on raw RGB data sampled from a square patch of the source image, centered on the pixel itself. The DNN is trained to differentiate patches with a mitotic nucleus close to the center from all other windows.

\section{CHALLENGES:}

Unlike fluorescence imaging, phase contrast microscopy is a form of nondestructive imaging method. Under phase con-trast microscope, cells can be monitored alive and continu-ously without changing their structures. Therefore, automated systems can be employed to observe and analyze cell behaviors by recording time-lapse images [1].

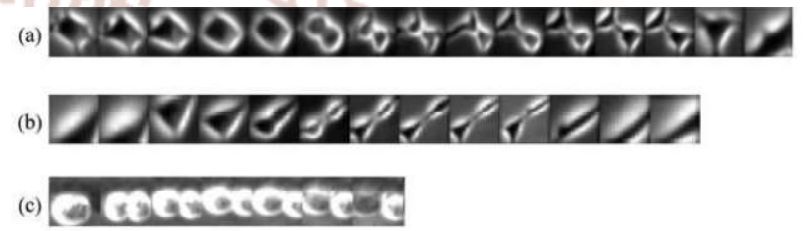

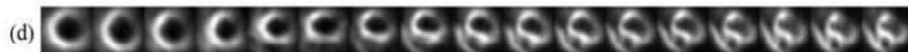

Fig 3 (a-c) The dynamic process of mitosis in phase contrast images.

(d) shows a non-mitotic sequences

operates on raw pixel values, no human input is

Different from other pattern recognition tasks, most mitotic cells have irregular shapes and appearance, as shown in Figs. 3a, 3b, and 3c. Samples may vary in orientations (Figs. $3 a$ and $3 b$ ), in appearances (Fig. 3b in $\mathrm{C} 3 \mathrm{H} 10$ dataset and Fig. 3c in $\mathrm{C} 2 \mathrm{C} 12$ dataset), and in temporal lengths (Figs. 3a, 3b, and 3c).

Moreover, some non-mitotic sequences may have similar appearance as mitosis sequences (Fig. 3d), which might cause false positives in detection. Therefore it is not sufficient to simply search for the shape ' 8 ' through phase contrast images to detect mitosis. Dense cell population data make mitosis detection challenging, and the level of difficulty only increases as live cells proliferate and further increase the population density where neighboring cells introduce ambiguity. Given their large number in a single microscopy image, it is difficult to find all of them by simply searching for a standard pattern. In recent years, many approaches have been proposed to solve this problem under different applications.

\section{EXISTING SYSTEM:}

Mitosis in unseen images are detected by applying the classifier on a sliding window, and post processing its outputs with simple techniques. Because the DNN needed : on the contrary, the DNN automatically learns a set of visual features from the training data. Our main contribution is a new, important, practical application of DNN, which recently produced outstanding results in image classification, segmentation and detection. Our approach is tested on a publicly available dataset.Our detector is a DNNbased pixel classifier. Windows of class mitosis contain a visible mitosis around the window's center. Others contain off-center or no mitosis.

Deep Neural Netwok architecture A DNN [4] is a feed-forward net made of successive pairs of convolutional and max-pooling layers, followed by several fully connected layers. Raw pixel intensities of the input image are passed through this general, hierarchical feature extractor. The feature vector it produces is classified by the fully connected layers. All weights are optimized through minimization of the misclassification error over the training set. Each convolutional layer performs a 2D convolution of its input maps with a rectangular filter. 


\section{CONCLUSION:}

In this paper we used four methods. Among all the four methods deep learning method is the best solution for detecting mitosis in phase contrast microscopy. Compared with hand crafted features, deep learning method could learn more discriminative and data relevant features directly from image data.

\section{REFERENCES}

1. F. Yang, M. A. Mackey, F. Ianzini, G. Gallardo, and M. Sonka, "Cell segmentation, tracking, and mitosis detection using tempo-ral context," in Proc. Int. Conf. Med. ImageComput. Comput.Assisted Intervention., 2005, pp. 302-309.

2. O. Debeir, P. Van Ham, R. Kiss, and C. Decaestecker, "Tracking of migrating cells phasecontrast video microscopy with com-bined meanshift processes," IEEE Trans. Med. Imag., vol. 24, no. 6,697-711, Jun. 2005.

3. K. Thirusittampalam, M. J. Hossain, O. Ghita, and P. F. Whelan, "A novel frameworkfor cellular tracking and mitosis detection in dense phase contrast microscopy images,'IEEE J. Biomed. Health Inform., vol. 17, no. 3, pp. 642-653, May. 2013.

4. A. Liu, T. Hao, Z. Gao, Y. Su, and Z. Yang, "Nonnegative mixed-norm convex optimization for mitotic cell detection in phase con-trast microscopy," Comput. Math. Methods Med., vol. 2013, Article ID 176272, p. 10, 2013, doi: $10.1155 / 2013 / 176272$.

5. M. Marcuzzo, T. Guichard, P. Quelhas, A. M. Mendonc,a, and A. Campilho, "Cell division detection on the arabidopsis thaliana root," in Proc. Iberian Conf. Pattern Recognition Image Anal., 2009,168-175.

6. G. M. Gallardo, F. Yang, F. Ianzini, M. Mackey, and M. Sonka, "Mitotic cell recognition with hidden markov models," in Med. Imag., vol. 5367, pp. 661-668, 2004.

7. L. M. Vincent and P. Soille, "Watersheds in digital spaces: An effi-cient algorithm based on immersion simulations," IEEE Trans. Pattern Anal. Mach. Intell., vol. 13, no. 6, pp. 583-598, Jun. 1991.

8. L. Liang, X. Zhou, F. Li, S. T. Wong, J. Huckins, and R. W. King, "Mitosis cell identification with conditional random fields," in Proc. Life Sci. Syst. Appl. Workshop, 2007, pp. 9-12.

9. D. C. Ciresan, , A. Giusti, L. M. Gambardella, and J. Schmidhuber, "Mitosis detection in breast cancer histology images with deep neural networks," in Proc. Int. Conf. Med. Image Comput. Comput.-Assisted Intervention, 2013, pp. 411-418. Y.

10. Mao and Z. Yin, "A hierarchical convolutional neural network for mitosis detection in phasecontrast microscopy images," inProc. Int. Conf. Med. Image Comput. Comput.-Assisted Intervention, 2016, pp. 685-692.

11. S. Huh, R. Bise, M. Chen, T. Kanade, D. F. E. Ker, "Automated mitosis detection of stem cell populations in phase-contrast microscopy images," IEEE Trans. Med. Imag., vol. 30, no. 3,586-596, Mar. 2011.

12. W. Nie, W. Li, A. Liu, T. Hao, and Y. Su, "3D convolutional networks-based mitotic event detection in time-lapse phase con-trast microscopy image sequences of stem cell populations," inProc. IEEE Conf. Comput. Vis. Pattern Recognition Workshops, 2016,55-62.

13. L. P. Morency, A. Quattoni, and T. Darrell, "Latent-dynamic dis-criminative models for continuous gesture recognition," in Proc. IEEE Conf. Comput. Vis. Pattern Recognition, 2007, pp. $1-8$.

14. P. Felzenszwalb, D. McAllester, and D. Ramanan, "A discrimina-tively trained, multiscale, deformable part model," in Proc. IEEE Conf. Comput. Vis. Pattern Recognition, 2008, pp. 1-8.

15. O. Al-Kofahi, R. Radke, S. Goderie, Q. Shen, S. Temple, and B. Roysam. Automated cell lineage construction: A rapid method to analyze clonal development established with murine neural progenitor cells. Cell Cycle, 5(3):327-335, 2006.

16. O. Debeir, P. V. Ham, R. Kiss, and C. Decaestecker. Tracking of mi-grating cells under phase-contrast video microscopy with combined mean-shift processes. IEEE Trans. Med. Imaging, 24(6):697-711, 2005.

17. O. Debeir, V. Megalizzi, N. Warzee, R. Kiss, and C. Decaestecker. Videomicroscopic extraction of specific information on cell prolif-eration and migration in virto. Exp. Cell Res., 314(16):29852998, 2008. 
18. A. Gunawardana, M. Mahajan, A. Acero, and J. Platt. Hidden con-ditional random fields for phone classification. In Proc. Interspeech, pages 11171120, 2005.

19. D. House, M. Walker, Z. Wu, J. Wong, and M. Betke. Tracking of cell populations to understand thier spatio-temporal behavior in response to physical stimuli. In Proc. IEEE Conference on Computer Vision and Pattern Recognition Workshop on Mathematical Methods in Biomedical Image Analysis, pages 186-193, 2009.

20. S. Huh, D. Ker, R. Bise, M. Chen, and T. Kanade. Automated mi-tosis detection of stem cell populations in phase-contrast microscopy images. IEEE Trans. Med. Imaging, 30(3):586-596, 2011.

21. T. Joachims. Making large-Scale SVM Learning Practical. Advances in Kernel Methods - Support Vector Learning. MIT-Press, 1999.

22. S. Kumar and M. Herbert. Discriminative random fields: A frame-work for contextual interaction in classification. In Proc. Interna-tional Conference on Computer Vision, pages 1150-1157, 2003.

23. Proc. International Conference on Machine Learning, pages 282-289, 2001. J. Pearl Probabilistic Reasoning in Intelligent Systems: Networks of Plausible Inference. Morgan Kaufmann, 1988.

24. H. Quastler and F. Sherman. Cell population kinetics in the intestinal epithelium of the mouse. Exp. Cell Res., 17(3):420-438, 1959.

25. A. Quattoni, S. Wang, L. Morency, M. Collins, and T. Darrell. Hid-den conditional random fields. IEEE Trans. Pattern Anal. Mach. In-tell., 29(10) 